\title{
Total Synthesis and Absolute Configuration of Otteliones A and B, Novel and Potent Antitumor Agents from a Freshwater Plant
}

\author{
- Supporting Information -
}

Hiroshi Araki, ${ }^{\dagger}$ Munenori Inoue, ${ }^{\ddagger}$ Tadashi Katoh ${ }^{*,+,}$

Department of Electronic Chemistry,

Tokyo Institute of Technology, Nagatsuta, Yokohama 226-8502, Japan and ${ }^{\ddagger}$ Sagami Chemical Research Center, 2743-1 Hayakawa, Ayase, Kanagawa 252-1193, Japan.

katoh@sagami.or.jp 


\section{General Procedure.}

All reactions involving air- and moisture-senstive reagent were carried out using oven dried glassware and standard syringe-septum cap techniques. Routine monitorings of reaction were carried out using glass-supported Merck silica gel $60 \mathrm{~F}_{254}$ TLC plates. Flash column chromatography was performed on Kanto Chemical Silica Gel 60N (spherical, neutral 40-50 $\mu \mathrm{m}$ ) with the solvents indicated.

\section{Materials.}

All solvents and reagent were used as supplied with the following exceptions. Tetrahydrofuran and diethyl ether were freshly distilled from sodium/benzophenone under argon.

\section{Instrumentation.}

Measurements of optical rotations were performed with a JASCO P-1020 automatic digital polarimeter. Melting points were taken on a Yanaco MP-3 micro melting point apparatus and are uncorrected. ${ }^{1} \mathrm{H}$ and ${ }^{13} \mathrm{C}$ NMR spectra were measured with a Brucker DRX-500 (500 MHz) spectrometer. Chemical shifts were expressed in ppm using tetramethylsilane $(\delta=0)$ as an internal standard. The following abbreviations are used: singlet $(\mathrm{s})$, doublet $(\mathrm{d})$, triplet $(\mathrm{t})$, multiplet $(\mathrm{m})$, and droad (br). Infrared (IR) spectral measurements were carried out with a JASCO FT/IR-5300 spectrometer. Low-resolution mass (MS) spectra was measured on a Shimadzu GCMS-QP2010. High-resolution mass (HRMS) spectra was measured on a JEOL MStation JMS-700 mass spectrometer. 
$(1 R, 4 S, 4 a R, 5 R, 6 R, 7 R, 8 S, 8 a S)$-5-tert-Butyldimethylsiloxy-8-hydroxy-6,7-O-isopropylidenedioxy1,4,4a,5,6,7,8,8a-octahydro-endo-1,4-methanonaphthalen-8-ol (7).

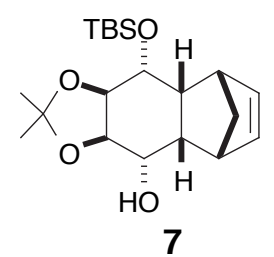

Sodium borohydride (153 mg, $4.0 \mathrm{mmol})$ was added to a stirred solution of $(1 R, 4 S, 4 \mathrm{a} S, 5 R, 6 S, 7 S, 8 \mathrm{a} S)$ 5-tert-butyldimethylsiloxy-6,7-O-isopropylidenedioxy-1,4,4a,5,6,7,8,8a-octahydro-endo-1,4-methanonaphthalen-8-one $(6)(737 \mathrm{mg}, 2.0 \mathrm{mmol})$ in $\mathrm{THF}-\mathrm{H}_{2} \mathrm{O}(22 \mathrm{ml}, 10: 1)$ at $0^{\circ} \mathrm{C}$. After $30 \mathrm{~min}$, the reaction was quenched with saturated aqueous ammonium chloride $(5 \mathrm{ml})$, and extracted with ether $(2 \mathrm{x} 100 \mathrm{ml})$. The organic layer was washed with saturated aqueous sodium hydrogen carbonate $(2 \times 50 \mathrm{ml})$ and brine $(2 \times 50 \mathrm{ml})$, then dried over $\mathrm{Na}_{2} \mathrm{SO}_{4}$. Concentration of the solvent in vacuo afforded a residue, which was purified by column chromatography (hexane-ethyl acetate, $10: 1 \rightarrow 4: 1)$ to give $7(643 \mathrm{mg}, 87 \%)$ as a white solid. Recrystallization from hexane-ether afforded colorless needles, mp $147.0-147.7^{\circ} \mathrm{C} ;[\alpha]_{\mathrm{D}}{ }^{20}-25.1^{\circ}(c$ 0.98, $\left.\mathrm{CHCl}_{3}\right) ;{ }^{1} \mathrm{H}$ NMR $\left(500 \mathrm{MHz}, \mathrm{CDCl}_{3}\right) \delta 0.10(6 \mathrm{H}, \mathrm{s}), 0.90(9 \mathrm{H}, \mathrm{s}), 1.23-1.34(4 \mathrm{H}, \mathrm{m}), 1.38-1.49(4 \mathrm{H}$, $\mathrm{m}), 2.58-2.79(2 \mathrm{H}, \mathrm{m}), 2.80-3.05(2 \mathrm{H}, \mathrm{m}), 3.80-4.30(4 \mathrm{H}, \mathrm{m}), 6.03(1 \mathrm{H}, \mathrm{s}), 6.09(1 \mathrm{H}, \mathrm{s}) ;{ }^{13} \mathrm{C}$ NMR $(125$ $\left.\mathrm{MHz}, \mathrm{CDCl}_{3}\right) \delta-4.95,-4.71,18.0,23.8,25.8,26.6,44.9,51.7,78.1,107.7,134.5$; IR (KBr) 3426, 2934, 2858, 2363, 1630, 1381, 1251, 1207, 1167, 1111, 1064, 898, 862, 837, $777 \mathrm{~cm}^{-1}$; CIMS m/z $367\left(\mathrm{M}+\mathrm{H}^{+}\right)$; Anal calcd for $\mathrm{C}_{20} \mathrm{H}_{34} \mathrm{O}_{4} \mathrm{Si}$ : C, 65.53; H, 9.35, found C, 65.57; H, 9.35.

$(2 S, 2 \mathrm{a} S, 4 R, 4 \mathrm{a} S, 5 R, 6 R, 7 R, 7 \mathrm{a} S, 7 \mathrm{~b} R)-5$-tert-Butyldimethylsiloxy-2-hydroxy-6,7-(O-isopropylidenedioxy)decahydroindeno[7,1-bc]furan-4-carbaldehyde (5).

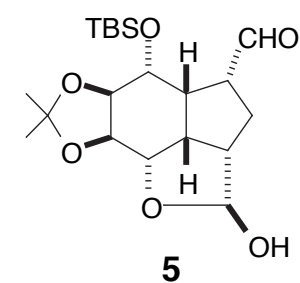

Osmium tetraoxide in tert-butyl alcohol $(0.04 \mathrm{M}$ solution, $1.30 \mathrm{ml}, 0.06 \mathrm{mmol})$ and sodium metaperiodate (467 mg, $2.2 \mathrm{mmol}$ ) were added successively to a stirred solution of $(1 R, 4 S, 4 \mathrm{a} R, 5 R, 6 R, 7 R, 8 S, 8 \mathrm{a} S)-5$-tertbutyldimethylsiloxy-8-hydroxy-6,7- $O$-isopropylidenedioxy-1,4,4a,5,6,7,8,8a-octahydro-endo-1,4methanonaphthalen-8-ol (7) $(200 \mathrm{mg}, 0.55 \mathrm{mmol})$ in tert-butyl alcohol-THF- $\mathrm{H}_{2} \mathrm{O}(10 \mathrm{ml}, 8: 6: 3)$ at $0^{\circ} \mathrm{C}$, and the mixture was allowed to warm up to room temperature. After $12 \mathrm{~h}$, the reaction was quenched with $10 \%$ aqueous sodium thiosulfate $(10 \mathrm{ml})$, and the resulting mixture was extracted with ether $(2 \times 50 \mathrm{ml})$. The combined extracts were washed with saturated aqueous sodium hydrogen carbonate ( 2 x $20 \mathrm{ml})$ and brine $(2 \times 20 \mathrm{ml})$, then dried over $\mathrm{Na}_{2} \mathrm{SO}_{4}$. Concentration of the solvent in vacuo afforded a residue, which was purified by column chromatography (hexane-ethyl acetate, 5:1) to give 5 (136 mg, 62\%) as a 
white solid. Recrystallization from hexane-ether afforded colorless needles, mp $120.7-121.5^{\circ} \mathrm{C} ;[\alpha]_{\mathrm{D}}{ }^{20}$ $+19.0^{\circ}\left(c\right.$ 0.99, $\left.\mathrm{CHCl}_{3}\right) ;{ }^{1} \mathrm{H}$ NMR $\left(500 \mathrm{MHz} \mathrm{CDCl}_{3}\right) \delta 0.06(3 \mathrm{H}, \mathrm{s}), 0.12(3 \mathrm{H}, \mathrm{s}), 0.88(9 \mathrm{H}, \mathrm{s}), 1.34(3 \mathrm{H}$, s), $1.44(3 \mathrm{H}, \mathrm{s}), 2.11(1 \mathrm{H}, \mathrm{m}), 2.22(1 \mathrm{H}, \mathrm{m}), 2.26(1 \mathrm{H}, \mathrm{d}, J=2.3 \mathrm{~Hz}), 2.74-2.91(3 \mathrm{H}, \mathrm{m}), 2.94(1 \mathrm{H}, \mathrm{m})$, $4.17(1 \mathrm{H}, \mathrm{dd}, J=6.7,4.5 \mathrm{~Hz}), 4.23(1 \mathrm{H}, \mathrm{s}), 4.47(1 \mathrm{H}, \mathrm{dd}, J=12.2,6.7 \mathrm{~Hz}), 4.47(1 \mathrm{H}, \mathrm{dd}, J=12.2,6.7 \mathrm{~Hz})$, $5.27(1 \mathrm{H}, \mathrm{d}, J=2.3 \mathrm{~Hz}), 9.93(1 \mathrm{H}, \mathrm{s}) ;{ }^{13} \mathrm{C} \mathrm{NMR}\left(125 \mathrm{MHz}, \mathrm{CDCl}_{3}\right) \delta-4.43,-4.37,18.2,24.3,25.9,27.0$, 30.2, 40.6, 41.9, 52.0, 55.8, 69.4, 73.6, 75.7, 76.0, 103.1, 107.7, 202.9; IR (KBr) 3433, 2955, 2928, 2854, 1714, 1469, 1371, 1246, 1221, 1151, 1103, 1057, 1012, 956, 898, 860, 831, 775, $511 \mathrm{~cm}^{-1}$; HREIMS m/z calcd for $\mathrm{C}_{20} \mathrm{H}_{34} \mathrm{O}_{6} \mathrm{Si}\left(\mathrm{M}^{+}\right), 398.2125$, found 398.2124.

$(2 S, 2 \mathrm{a} S, 4 R, 4 \mathrm{a} R, 5 R, 6 R, 7 R, 7 \mathrm{a} S, 7 \mathrm{~b} R)-5$-tert-Butyldimethylsiloxy-4-[hydroxy[4-methoxy-3(methoxymethoxy)phenyl]methyl]-6,7-( $O$-isopropylidenedioxy)decahydroindeno[7,1-bc]furan-2-ol (10a,b).

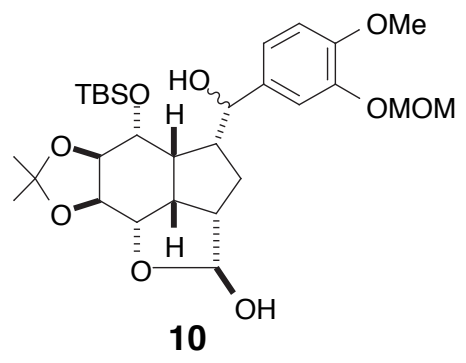

$n$-Butyllithium in $n$-hexane (1.55 M solution, $4.40 \mathrm{ml}, 7.0 \mathrm{mmol})$ was added dropwise to a stirred solution of 4-bromo-1-methoxy-2-(methoxymetoxy)benzene $(9)^{1}(2.30 \mathrm{~g}, 7.7 \mathrm{mmol})$ in dry $\mathrm{THF}(40 \mathrm{ml})$ at $-78^{\circ} \mathrm{C}$ under argon. After $1 \mathrm{~h}$, a solution of $(2 S, 2 \mathrm{a} S, 4 R, 4 \mathrm{a} S, 5 R, 6 R, 7 R, 7 \mathrm{a} S, 7 \mathrm{~b} R)$-5-tert-butyldimethylsiloxy-2hydroxy-6,7-( $O$-isopropylidenedioxy)decahydroindeno[7,1-bc]furan-4-carbaldehyde $\quad(5) \quad(930 \quad \mathrm{mg}, \quad 2.3$ $\mathrm{mmol})$ in dry $\mathrm{THF}(30 \mathrm{ml})$ was added to the above mixture at $-78^{\circ} \mathrm{C}$. After $1 \mathrm{~h}$, the reaction was quenched with saturated aqueous ammonium chloride $(10 \mathrm{ml})$, and the resulting mixture was extracted with ether $(2$ x $100 \mathrm{ml})$. The organic layer was washed with saturated aqueous sodium hydrogen carbonate $(2 \times 30 \mathrm{ml})$ and brine $(30 \mathrm{ml})$, then dried over $\mathrm{Na}_{2} \mathrm{SO}_{4}$. Concentration of the solvent in vacuo afforded a residue, which was purified by column chromatography (hexane-ethyl acetate, $2: 1 \rightarrow 1: 1)$ to give 10a $(1.06 \mathrm{~g}$, $80 \%$ ) (more polar) and $\mathbf{1 0 b}(263 \mathrm{mg}, 20 \%)$ (less polar).

10a: colorless oil; $[\alpha]_{\mathrm{D}}{ }^{20}+20.1^{\circ}\left(c 0.74, \mathrm{CHCl}_{3}\right) ;{ }^{1} \mathrm{H} \mathrm{NMR}\left(500 \mathrm{MHz}, \mathrm{CDCl}_{3}\right) \delta 0.17(3 \mathrm{H}, \mathrm{s}), 0.24(3 \mathrm{H}$, s), $0.95(9 \mathrm{H}, \mathrm{s}), 1.26(6 \mathrm{H}, \mathrm{s}), 1.81(1 \mathrm{H}, \mathrm{br}), 1.88(1 \mathrm{H}, \mathrm{br}), 1.97-2.10(1 \mathrm{H}, \mathrm{m}), 2.27(1 \mathrm{H}, \mathrm{br}), 2.29(1 \mathrm{H}, \mathrm{d}$, $J=2.4 \mathrm{~Hz}), 2.47-2.57(1 \mathrm{H}, \mathrm{m}), 2.76-2.87(2 \mathrm{H}, \mathrm{m}), 3.51(3 \mathrm{H}, \mathrm{s}), 3.88(3 \mathrm{H}, \mathrm{s}), 3.99(1 \mathrm{H}, \mathrm{t}, J=3.4 \mathrm{~Hz}), 4.18-$ $4.27(1 \mathrm{H}, \mathrm{m}), 4.41-4.45(1 \mathrm{H}, \mathrm{m}), 4.47(1 \mathrm{H}, \mathrm{dd}, \mathrm{J}=1.6,7.1 \mathrm{~Hz}), 4.81-4.91(1 \mathrm{H}, \mathrm{m}), 5.23(2 \mathrm{H}, \mathrm{s}), 5.27(1 \mathrm{H}$, $\mathrm{d}, J=2.5 \mathrm{~Hz}), 6.88(1 \mathrm{H}, \mathrm{d}, J=8.3 \mathrm{~Hz}), 7.03(1 \mathrm{H}, \mathrm{dd}, J=8.3,1.9 \mathrm{~Hz}), 7.22(1 \mathrm{H}, \mathrm{d}, J=1.9 \mathrm{~Hz}) ;{ }^{13} \mathrm{C}$ NMR $(125$ $\left.\mathrm{MHz}, \mathrm{CDCl}_{3}\right) \delta-4.07,-3.82,18.5,23.7,26.1,26.4,32.7,36.6,42.1,52.1,52.4,53.4,55.9,56.1,68.6$, 73.9, 74.9, 76.0, 95.6, 103.6, 107.4, 111.7, 114.9, 120.5, 136.6, 146.7, 149.4; IR (neat) 3423, 2932, 2856, $1606,1510,1464,1385,1259,1211,1155,1132,1080,1012,868,835,775 \mathrm{~cm}^{-1}$; HREIMS m/z calcd for $\mathrm{C}_{29} \mathrm{H}_{46} \mathrm{O}_{9} \mathrm{Si}\left(\mathrm{M}^{+}\right)$, 566.2911, found 566.2904.

10b: colorless oil; $[\alpha]_{\mathrm{D}}{ }^{20}+12.5^{\circ}\left(c 0.74, \mathrm{CHCl}_{3}\right) ;{ }^{1} \mathrm{H}$ NMR $\left(500 \mathrm{MHz}, \mathrm{CDCl}_{3}\right) \delta 0.20(3 \mathrm{H}, \mathrm{s}), 0.24(3 \mathrm{H}$, s), $0.97(9 \mathrm{H}, \mathrm{s}), 1.26(3 \mathrm{H}, \mathrm{s}), 1.38-1.42(1 \mathrm{H}, \mathrm{m}), 1.44(3 \mathrm{H} . \mathrm{s}), 1.67-1.76(1 \mathrm{H}, \mathrm{m}), 1.77(1 \mathrm{H}, \mathrm{s}), 2.16(1 \mathrm{H}$, s), 2.33-2.42 (1H, m), 2.50-2.58 (1H, m), 2.63-2.72 (1H, m), 2.84-2.92 (1H, m), 3.51 (3H. s), $3.87(3 \mathrm{H}, \mathrm{s})$, 
4.35-4.40 (1H, m), $4.48(1 \mathrm{H}, \mathrm{d}, \mathrm{J}=7.0 \mathrm{~Hz}), 4.54(1 \mathrm{H}, \mathrm{d}, J=6.4 \mathrm{~Hz}), 4.53-4.59(1 \mathrm{H}, \mathrm{m}), 4.86$ (1H, d, $J=10.7$ $\mathrm{Hz}), 5.18(1 \mathrm{H}, \mathrm{s}), 5.21(2 \mathrm{H}, \mathrm{s}), 6.87(1 \mathrm{H}, \mathrm{d}, J=8.3 \mathrm{~Hz}), 6.97(1 \mathrm{H}, \mathrm{d}, J=7.7 \mathrm{~Hz}), 7.19(1 \mathrm{H}, \mathrm{s}) ;{ }^{13} \mathrm{C}$ NMR $\left(125 \mathrm{MHz}, \mathrm{CDCl}_{3}\right) \delta-3.81,-3.66,18.5,23.7,25.8,26.2,26.5,32.6,36.4,42.0,52.6,53.1,68.3,72.4$, 73.8, 74.5, 75.1, 95.6, 103.8, 107.5, 111.6, 114.8, 120.0, 137.6, 146.4, 149.3; IR (neat) 3435, 2934, 1510, $1464,1383,1261,1157,1078,1049,1006,868,835,775 \mathrm{~cm}^{-1} ;$ HRFABMS m/z calcd for $\mathrm{C}_{29} \mathrm{H}_{46} \mathrm{O}_{9} \mathrm{Si}$ $\left(\mathrm{M}^{+}\right)$, 566.2911, found 566.2914.

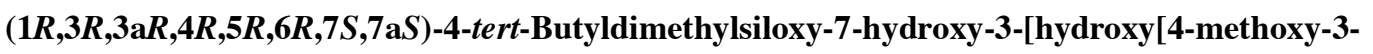
(methoxymethoxy)phenyl]methyl]-5,6-( $O$-isopropylidenedioxy)octahydroindene-1-carbaldehyde (11).

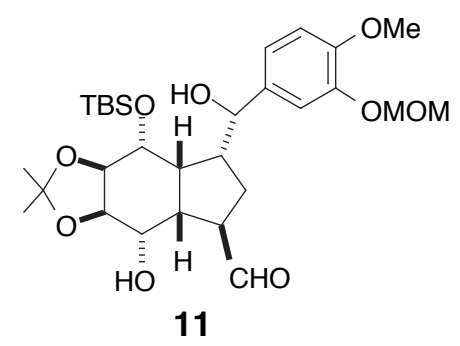

1,8-Diazabicyclo[5.4.0] undec-7-ene (DBU) $(4.70 \mathrm{~mL}, 32 \mathrm{mmol})$ was added to a stirred solution of $(2 S, 2 \mathrm{a} S, 4 R, 4 \mathrm{a} R, 5 R, 6 R, 7 R, 7 \mathrm{a} S, 7 \mathrm{~b} R)$-5-tert-butyldimethylsiloxy-4-[hydroxy[4-methoxy-3-(methoxymethoxy)phenyl]methyl]-6,7-( $O$-isopropylidenedioxy)decahydroindeno[7,1- $b c$ furan-2-ol (10a) (900 mg, 1.6 $\mathrm{mmol})$ in dry toluene $(32 \mathrm{ml})$ at room temperature under argon. The mixture was heated at reflux for $1.5 \mathrm{~h}$. After cooling, the mixture was directly subjected to column chromatography (ethyl acetate) in order to remove DBU. The fractions containing 11 and starting material 10a were collected and then concentrated in vacuo. The residue was purified by column chromatography (hexane-ethyl acetate, $2: 1 \rightarrow 1: 1$ ) to give less polar 11 (270 mg, 30\%) (colorless oil) and more polar starting material 10a (540 mg, $60 \%$ ). 11: $[\alpha]_{\mathrm{D}}{ }^{20}-34.3^{\circ}\left(c 0.98, \mathrm{CHCl}_{3}\right) ;{ }^{1} \mathrm{H}$ NMR $\left(500 \mathrm{MHz}, \mathrm{CDCl}_{3}\right) \delta 0.23(3 \mathrm{H}, \mathrm{s}), 0.31(3 \mathrm{H}, \mathrm{s}), 0.97(9 \mathrm{H}, \mathrm{s})$, 2.00-2.12 (1H, m), 2.22-2.43 (3H, m), 3.01-3.09 (1H, m), 3.35-3.44 (1H, m), $3.50(3 \mathrm{H}, \mathrm{s}), 3.81-3.86(1 \mathrm{H}$, m), $3.87(3 \mathrm{H}, \mathrm{s}), 4.02(1 \mathrm{H}, \mathrm{ddd}, J=4.1,6.6,10.8 \mathrm{~Hz}), 4.14-4.22(1 \mathrm{H}, \mathrm{m}), 4.45(1 \mathrm{H}, \mathrm{dr}), 4.73(1 \mathrm{H}, \mathrm{dr}), 6.87$ $(1 \mathrm{H}, \mathrm{d}, J=8.3 \mathrm{~Hz}), 6.99(1 \mathrm{H}, \mathrm{dd}, J=2.0,8.3 \mathrm{~Hz}), 7.18,(1 \mathrm{H}, \mathrm{d}, J=2.0 \mathrm{~Hz}) ;{ }^{13} \mathrm{C} \mathrm{NMR}\left(125 \mathrm{MHz}, \mathrm{CDCl}_{3}\right) \delta$ $-4.33,-3.84,14.2,18.1,21.0,23.8,25.9,26.1,50.7,55.9,56.2,60.3,75.7,75.9,77.2,95.6,108.5,111.8$, 114.9, 120.1, 135.9, 146.7, 203.0; IR (neat) 3422, 2934, 2860, 1718, 1512, 1383, 1261, 1211, 1155, 1078, 1022, 864, 839, $781 \mathrm{~cm}^{-1}$; HREIMS m/z calcd for $\mathrm{C}_{29} \mathrm{H}_{46} \mathrm{O}_{9} \mathrm{Si}\left(\mathrm{M}^{+}\right)$, 566.2911, found 566.2916. 
$(1 R, 3 R, 3 \mathrm{a} S, 4 S, 5 R, 6 R, 7 R, 7 \mathrm{a} R)$-7-tert-Butyldimethylsiloxy-1-[hydroxy[4-methoxy-3(methoxymethoxy)phenyl]methyl]-3-hydroxymethyl-5,6-( $O$-isopropylidenedioxy)octahydroindene4-ol (12).

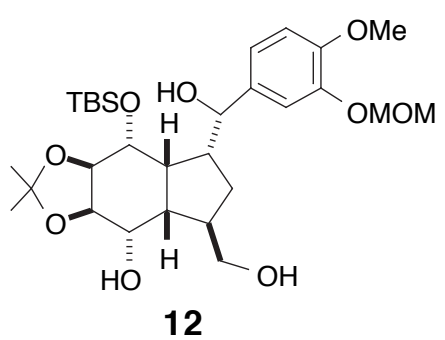

A solution of $(1 R, 3 R, 3 \mathrm{a} R, 4 R, 5 R, 6 R, 7 S, 7 \mathrm{a} S)$-4-tert-butyldimethylsiloxy-7-hydroxy-3-[hydroxy[4methoxy-3-(methoxymethoxy)phenyl]methyl]-5,6-( $O$-isopropylidenedioxy)octahydroindene-1-

carbaldehyde (11) (306 mg, $0.54 \mathrm{mmol}$ ) in dry THF (6 ml) was added dropwise to a stirred suspention of lithium aluminium hydride $(41.0 \mathrm{mg}, 1.1 \mathrm{mmol})$ in dry tetrahydrofuran $(4 \mathrm{ml})$ at $0^{\circ} \mathrm{C}$ under argon. After 1 min, water $(41 \mu \mathrm{l}), 15 \%$ aqueous sodium hydroxide $(41 \mu \mathrm{l})$, water $(123 \mu \mathrm{l})$, and $\mathrm{Na}_{2} \mathrm{SO}_{4}(500 \mathrm{mg})$ were added successively to the mixture, and the resulting mixture was stirred for $30 \mathrm{~min}$ at room temperature. The mixture was filtrated through a pad of Celite ${ }^{\circledR}$, and the filtrate was concentrated in vacuo to afford a residue, which was purified by column chromatography (hexane-ethyl acetate, 1:1 $\rightarrow$ ethyl acetate) to give $12(294 \mathrm{mg}, 96 \%)$ as a colorless oil: $[\alpha]_{\mathrm{D}}{ }^{20}-36.6^{\circ}\left(c 0.83, \mathrm{CHCl}_{3}\right) ;{ }^{1} \mathrm{H} \mathrm{NMR}\left(500 \mathrm{MHz}, \mathrm{CDCl}_{3}\right) \delta 0.22$ $(3 \mathrm{H}, \mathrm{s}), 0.96(3 \mathrm{H}, \mathrm{s}), 1.26(6 \mathrm{H}, \mathrm{s}), 1.63(1 \mathrm{H}, \mathrm{br}), 1.74(1 \mathrm{H}, \mathrm{br}), 1.90-2.00(1 \mathrm{H}, \mathrm{m}), 2.31-2.39(2 \mathrm{H}, \mathrm{m})$, 2.46-2.55 (1H, m), $2.68(1 \mathrm{H}, \mathrm{br}), 3.39-3.47(1 \mathrm{H}, \mathrm{m}), 3.51(3 \mathrm{H}, \mathrm{s}), 3.48-3.56(1 \mathrm{H}, \mathrm{m}), 3.56-3.63(1 \mathrm{H}, \mathrm{m})$, 3.83-3.90 (1H, m), $3.87(3 \mathrm{H}, \mathrm{s}), 4.04(1 \mathrm{H}, \mathrm{br}), 4.17-4.23(1 \mathrm{H}, \mathrm{m}), 4.42(1 \mathrm{H}, \mathrm{dd}, J=5.2,7.3 \mathrm{~Hz}), 4.81-4.86$ $(1 \mathrm{H}, \mathrm{m}), 5.23(2 \mathrm{H}, \mathrm{s}), 6.87(1 \mathrm{H}, \mathrm{d}, J=8.3 \mathrm{~Hz}), 7.01(1 \mathrm{H}, \mathrm{dd}, J=2.0,8.3 \mathrm{~Hz}), 7.19(1 \mathrm{H}, \mathrm{d}, J=2.0 \mathrm{~Hz}) ;{ }^{13} \mathrm{C}$ NMR $\left(125 \mathrm{MHz}, \mathrm{CDCl}_{3}\right) \delta-4.31,-3.99,18.1,24.0,25.9,26.1,26.2,30.2,40.0,43.7,49.0,55.9,56.2$, 67.3, 67.4, 72.0, 73.0, 76.4, 76.5, 95.5, 108.5, 111.5, 114.5, 120.0, 136.4, 146.5, 149.1; IR (neat) 3435, 2932, 1512, 1383, 1259, 1155, 1078, 1018, 864, 837., $779 \mathrm{~cm}^{-1}$; HREIMS m/z calcd for $\mathrm{C}_{29} \mathrm{H}_{48} \mathrm{O}_{9} \mathrm{Si}\left(\mathrm{M}^{+}\right)$, 568.3068 , found 568.3085 .

$(1 R, 3 R, 3 \mathrm{a} S, 4 S, 5 R, 6 R, 7 R, 7 \mathrm{a} R)-4-A c e t o x y-3$-acetoxymethyl-7-tert-butyldimethylsiloxy-1-[acetoxy[4methoxy-3-(methoxymethoxy)phenyl]methyl]-5,6-(O-isopropylidenedioxy)octahydroindene (13).

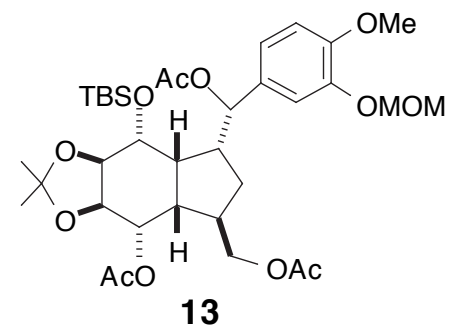

Acetic anhydride $(1.50 \mathrm{ml}, 16 \mathrm{mmol})$ and 4-dimethylaminopyridine (DMAP) (19.0 mg, $0.16 \mathrm{mmol})$ were added to a stirred solution of $(1 R, 3 R, 3 \mathrm{a} S, 4 S, 5 R, 6 R, 7 R, 7 \mathrm{a} R)$-7-tert-butyldimethylsiloxy-1-[hydroxy[4methoxy-3-(methoxymethoxy)phenyl]methyl]-3-hydroxymethyl-5,6-( $O$-isopropylidenedioxy)octahydro- 
indene-4-ol (12) (294 mg, $0.52 \mathrm{mmol})$ in pyridine $(5 \mathrm{ml})$ at room temperature. After $1 \mathrm{~h}$, the mixture was diluted with ether $(50 \mathrm{ml})$. The organic layer was washed with $3 \%$ hydrochloric acid $(2 \times 10 \mathrm{ml})$, saturated aqueous sodium hydrogen carbonate $(2 \times 10 \mathrm{ml})$, and brine $(10 \mathrm{ml})$, then dried over $\mathrm{Na}_{2} \mathrm{SO}_{4}$. Concentration of the solvent in vасио gave a residue, which was purified by column chromatography (hexane-ethyl acetate, 1:1) to give $13(324 \mathrm{mg}, 95 \%)$ as a colorless oil: $[\alpha]_{\mathrm{D}}{ }^{20}-38.8^{\circ}\left(c 1.16, \mathrm{CHCl}_{3}\right) ;{ }^{1} \mathrm{H}$ NMR $\left(500 \mathrm{MHz}, \mathrm{CDCl}_{3}\right) \delta 0.20(3 \mathrm{H}, \mathrm{s}), 0.33(3 \mathrm{H}, \mathrm{s}), 0.96(9 \mathrm{H}, \mathrm{s}), 1.13(3 \mathrm{H}, \mathrm{s}), 1.21(3 \mathrm{H}, \mathrm{s}), 1.62(1 \mathrm{H}$, ddd, $J=3.8,8.6,12.6 \mathrm{~Hz}), 1.99(3 \mathrm{H}, \mathrm{s}), 2.03(3 \mathrm{H}, \mathrm{s}), 2.09(3 \mathrm{H}, \mathrm{s}), 1.96-2.13(2 \mathrm{H}, \mathrm{m}), 2.31-2.37(1 \mathrm{H}, \mathrm{m})$, 2.66-2.75 (1H, m), 2.76-2.85 (1H, m), $3.51(3 \mathrm{H}, \mathrm{s}), 3.86(3 \mathrm{H}, \mathrm{s}), 3.84-3.89(1 \mathrm{H}, \mathrm{m}), 3.94-4.03(2 \mathrm{H}, \mathrm{m})$, $4.06(1 \mathrm{H}, \mathrm{dd}, J=2.4,6.3 \mathrm{~Hz}), 4.21(1 \mathrm{H}, \mathrm{dd}, J=4.9,6.3 \mathrm{~Hz}), 5.20-5.26(2 \mathrm{H}, \mathrm{m}), 5.43(1 \mathrm{H}, \mathrm{dd}, J=4.7,8.4$ $\mathrm{Hz}), 5.82(1 \mathrm{H}, \mathrm{d}, J=10.6 \mathrm{~Hz}), 6.86(1 \mathrm{H}, \mathrm{d}, J=8.3 \mathrm{~Hz}), 7.05(1 \mathrm{H}, \mathrm{dd}, J=1.9,8.3 \mathrm{~Hz}), 7.23(1 \mathrm{H}, \mathrm{d}, J=1.9$ $\mathrm{Hz}) ;{ }^{13} \mathrm{C}$ NMR $\left(125 \mathrm{MHz}, \mathrm{CDCl}_{3}\right) \delta-3.96,-3.89,18.1,20.9,21.2,24.7,26.0,26.4,31.9,37.3,39.7,41.8$, 46.0, 55.9, 56.2, 68.2, 68.8, 69.3, 73.5, 76.6, 77.1, 95.6, 108.1, 111.3, 115.4, 121.7, 132.5, 146.5, 149.6, 169.9, 170.4, 171.3; IR (neat) 2935, 2856, 1738, 1608, 1516, 1466, 1371, 1238, 1157, 1371, 1238, 1157 , 1132, 1078, 1024, 869, 837, 775, 607, 518, $412 \mathrm{~cm}^{-1}$; HREIMS m/z calcd for $\mathrm{C}_{35} \mathrm{H}_{54} \mathrm{O}_{12} \mathrm{Si}\left(\mathrm{M}^{+}\right), 694.3385$, found 694.3380 .

$(1 S, 3 R, 3 \mathrm{a} S, 4 S, 5 R, 6 R, 7 R, 7 \mathrm{a} R)$-7-tert-Butyldimethylsiloxy-3-hydroxymethyl-5,6- $O$-isopropylidenedioxy-1-[4-methyoxy-3-(methoxymethoxy)benzyl]octahydroindene-4-ol (14).

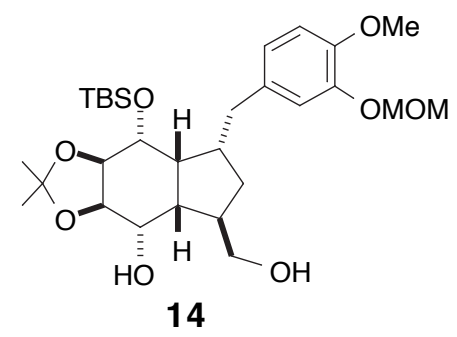

A solution of $(1 R, 3 R, 3 \mathrm{a} S, 4 S, 5 R, 6 R, 7 R, 7 \mathrm{a} R)$-4-acetoxy-3-acetoxymethyl-7-tert-butyldimethylsiloxy-1[acetoxy[4-methoxy-3-(methoxymethoxy)phenyl]methyl]-5,6- $(O$-isopropylidenedioxy)octahydroindene (13) $(340 \mathrm{mg}, 0.49 \mathrm{mmol})$ in dry THF (15 ml) was added dropwise to a stirred solution of lithium (100 $\mathrm{mg}, 9.0 \mathrm{mmol})$ in liquid ammonia $(30 \mathrm{ml})$ at $-78^{\circ} \mathrm{C}$ under argon. After $5 \mathrm{~min}$, the reaction was quenched with saturated aqueous ammonium chloride $(5 \mathrm{ml})$ at the same temperature. The mixture was then allowed to stand at room temperature for $4 \mathrm{~h}$ in order to evaporate off ammonia. The mixture was extracted with ethyl acetate $(2 \times 100 \mathrm{ml})$, and the extracts were washed successively with saturated aqueous sodium hydrogen carbonate $(2 \times 50 \mathrm{ml})$ and brine $(50 \mathrm{ml})$, then dried over $\mathrm{Na}_{2} \mathrm{SO}_{4}$. Concentration of the solvent in vacuo afforded a residue, which was purified by column chromatography (hexane-ethyl acetate, $1: 1)$ to give $14(265 \mathrm{mg}, 98 \%)$ as a colorless oil: $[\alpha]_{\mathrm{D}}{ }^{20}-27.7^{\circ}\left(c 0.91, \mathrm{CHCl}_{3}\right) ;{ }^{1} \mathrm{H}$ NMR $\left(500 \mathrm{MHz}, \mathrm{CDCl}_{3}\right) \delta 0.23(3 \mathrm{H}, \mathrm{s}), 0.26(3 \mathrm{H}, \mathrm{s}), 0.97(9 \mathrm{H}, \mathrm{s}), 1.34(3 \mathrm{H}, \mathrm{s}), 1.44(3 \mathrm{H}, \mathrm{s}), 1.43-1.49$ $(1 \mathrm{H}, \mathrm{m}), 1.63-1.72(1 \mathrm{H}, \mathrm{m}), 1.81(1 \mathrm{H}, \mathrm{br}), 2.26-2.38(2 \mathrm{H}, \mathrm{m}), 2.50-2.57(2 \mathrm{H}, \mathrm{m}), 2.60-2.69(1 \mathrm{H}, \mathrm{m}), 2.88$ $(1 \mathrm{H}, \mathrm{dd}, J=4.6,13.3 \mathrm{~Hz}), 3.43(1 \mathrm{H}, \mathrm{dd}, J=7.3,10.3 \mathrm{~Hz}), 3.47-3.55(1 \mathrm{H}, \mathrm{m}), 3.52(3 \mathrm{H}, \mathrm{s}), 3.86(3 \mathrm{H}, \mathrm{s})$, 3.90-4.04 (2H, m), $4.06(1 \mathrm{H}, \mathrm{t}, \mathrm{J}=3.0 \mathrm{~Hz}), 4.28(1 \mathrm{H}, \mathrm{dd}, J=2.9,7.2 \mathrm{~Hz}), 4,50-4.55(1 \mathrm{H}, \mathrm{m}), 5.19-5.23(2 \mathrm{H}$, m), $6.77(1 \mathrm{H}, \mathrm{dd}, J=1.9,8.2 \mathrm{~Hz}), 6.82(1 \mathrm{H}, \mathrm{d}, J=8.2 \mathrm{~Hz}), 6.99(1 \mathrm{H}, \mathrm{d}, J=1.9 \mathrm{~Hz}) ;{ }^{13} \mathrm{C} \mathrm{NMR}(125 \mathrm{MHz}$, 
$\left.\mathrm{CDCl}_{3}\right) \delta-4.46,-3.56,18.1,24.0,25.9,26.4,33.8,35.4,40.1,40.6,42.4,42.8,55.9,56.1,67.1,67.3$, 71.6, 76.1, 76.3, 95.5, 108.4, 111.6, 116.9, 122.1, 134.3, 146.3, 147.8; IR (neat) 3433, 2932, 2858, 1512, 1466, 1383, 1261, 1211, 1155, 1134, 1078, 1016, 923, 868, 837, 808, $779 \mathrm{~cm}^{-1}$; HREIMS m/z calcd for $\mathrm{C}_{29} \mathrm{H}_{48} \mathrm{O}_{8} \mathrm{Si}\left(\mathrm{M}^{+}\right)$, 552.3118, found 552.3129.

$(1 R, 3 S, 3 \mathrm{a} R, 4 R, 5 R, 6 S, 7 \mathrm{a} R)$-4-tert-Butyldimethylsiloxy-5,6-O-isopropylidenedioxy-3-[4-methoxy-3(methoxymethoxy)benzyl]-7-oxooctahydroindene-1-carbaldehyde (15).

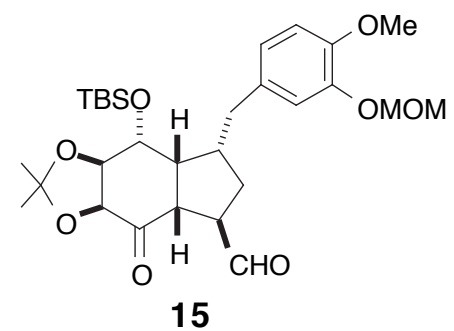

Dess-Martin periodinane $(609 \mathrm{mg}, 1.4 \mathrm{mmol})$ was added to a stirred solution of $(1 S, 3 R, 3 \mathrm{a} S, 4 S, 5 R, 6 R, 7 R, 7 \mathrm{a} R)$-7-tert-butyldimethylsiloxy-3-hydroxymethyl-5,6- $O$-isopropylidenedioxy-1[4-methyoxy-3-(methoxymethoxy)benzyl]octahydroindene-4-ol (14) (265 $\mathrm{mg}, 0.48 \mathrm{mmol}$ ) in dry dichloromethane $(10 \mathrm{ml})$ at room temperature. After $30 \mathrm{~min}$, the reaction was quenched with $10 \%$ aqueous sodium thiosulfate $(3 \mathrm{ml})$, and the resulting mixture was extracted with ether $(2 \times 25 \mathrm{ml})$. The organic layer was washed with saturated aqueous sodium hydrogen carbonate $(2 \mathrm{x} 10 \mathrm{ml})$ and brine $(10$ $\mathrm{ml}$ ), then dried over $\mathrm{Na}_{2} \mathrm{SO}_{4}$. Concentration of the solvent in vacuo afforded a residue, which was purified by column chromatography (hexane-ethyl acetate, 6:1) to give $15(210 \mathrm{mg}, 80 \%)$ as a colorless oil: $[\alpha]_{\mathrm{D}}{ }^{20}$ $+24.5^{\circ}\left(\mathrm{c} 2.50, \mathrm{CHCl}_{3}\right) ;{ }^{1} \mathrm{H} \mathrm{NMR}\left(500 \mathrm{MHz}, \mathrm{CDCl}_{3}\right) \delta 0.16(3 \mathrm{H}, \mathrm{s}), 0.22(3 \mathrm{H}, \mathrm{s}), 0.86(9 \mathrm{H}, \mathrm{s}), 1.34(3 \mathrm{H}$, s), $1.57(3 \mathrm{H}, \mathrm{s}), 1.54-1.92(2 \mathrm{H}, \mathrm{m}), 2.13-2.23(1 \mathrm{H}, \mathrm{m}), 2.57(1 \mathrm{H}, \mathrm{dd}, J=10.0,13.5 \mathrm{~Hz}), 2.81(1 \mathrm{H}, \mathrm{dd}$, $J=5.3,13.5 \mathrm{~Hz}), 3.13-3.19(1 \mathrm{H}, \mathrm{m}), 3.47-3.51(3 \mathrm{H}, \mathrm{s}), 3.52(3 \mathrm{H}, \mathrm{s}), 3.68(1 \mathrm{H}, \mathrm{dd}, J=5.1,9.6 \mathrm{~Hz}), 4.09-$ $4.11(1 \mathrm{H}, \mathrm{m}), 4.33(1 \mathrm{H}, \mathrm{d}, J=7.4 \mathrm{~Hz}), 4.40(1 \mathrm{H}, \mathrm{dd}, J=3.1,7.4 \mathrm{~Hz}), 5.21(2 \mathrm{H}, \mathrm{s}), 6.75(1 \mathrm{H}, \mathrm{dd}, J=1.9,8.2$ $\mathrm{Hz}), 6.82(1 \mathrm{H}, \mathrm{d}, J=8.2 \mathrm{~Hz}), 6.97(1 \mathrm{H}, \mathrm{d}, J=1.9 \mathrm{~Hz}) ;{ }^{13} \mathrm{C} \mathrm{NMR}\left(125 \mathrm{MHz}, \mathrm{CDCl}_{3}\right) \delta-4.07,-3.56,17.9$, 24.0, 25.6, 25.9, 30.5, 35.2, 43.3, 44.1, 44.8, 52.1, 55.9, 56.1, 68.8, 77.2, 80.4, 95.5, 111.7, 112.0, 116.7, 121.9, 133.2, 146.4, 148.0, 201.9, 206.2; IR (neat) 2932, 2858, 1728, 1514, 1466, 1383, 1263, 1209, 1157 , 1134, 1060, 1006, 925, 858, 837, 808, 777, 538, 468, $405 \mathrm{~cm}^{-1}$; HREIMS m/z calcd for $\mathrm{C}_{29} \mathrm{H}_{44} \mathrm{O}_{8} \mathrm{Si}\left(\mathrm{M}^{+}\right)$, 548.2805 , found 548.2801 .

$(1 S, 3 S, 3 a S, 5 R, 6 R, 7 R, 7 \mathrm{a} R)$-7-tert-Butyldimethylsiloxy-5,6-O-isopropylidenedioxy-1-[4-methoxy-3(methoxymethoxy)benzyl]-4-methylene-3-(vinyl)octahydroindene (3).

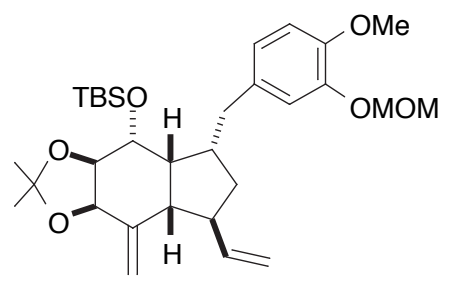


Wittig reagent (methylene triphenylphosphorane) in benzene solution was first prepared as follows: a suspension of methyl triphenylphosphonium bromide $(1.22 \mathrm{~g}, 3.4 \mathrm{mmol}$ ) and potassium tert-butoxide (380 $\mathrm{mg}, 3.4 \mathrm{mmol})$ in dry benzene $(10 \mathrm{ml})$ were heated at reflux for $4 \mathrm{~h}$ under argon, and the solution was cooled to room temperature. A solution of the Wittig reagent in benzene $(1.0 \mathrm{ml}, 0.34 \mathrm{mmol})$ was added very slowly to a stirred solution of $(1 R, 3 S, 3 \mathrm{a} R, 4 R, 5 R, 6 \mathrm{~S}, 7 \mathrm{a} R)-4$-tert-butyldimethylsiloxy-5,6-Oisopropylidenedioxy-3-[4-methoxy-3-(methoxymethoxy)benzyl]-7-oxooctahydroindene-1-carbaldehyde (15) $(187 \mathrm{mg}, 0.34 \mathrm{mmol})$ in dry benzene $(8 \mathrm{ml})$ at room temperature under argon. After the first olefination of the formyl group was completed (monitored by TLC), a solution of the Wittig reagent in benzene $(4.0 \mathrm{ml}, 1.36 \mathrm{mmol})$ was added once again and the resulting mixture was refluxed for 30 min to pursue the second olefination of the carbonyl group. After cooling, the reaction was quenched with water $(2 \mathrm{ml})$, and the mixture was extracted with ether $(2 \times 30 \mathrm{ml})$. The organic layer was washed with brine (10 $\mathrm{ml}$ ), then dried over $\mathrm{Na}_{2} \mathrm{SO}_{4}$. Concentration of the solvent in vacuo afforded a residue, which was purified by column chromatography (hexane-ethyl acetate, $20: 1 \rightarrow 10: 1)$ to give $\mathbf{3}(177 \mathrm{mg}, 95 \%$ ) as a colorless oil: $[\alpha]_{\mathrm{D}}{ }^{20}-21.9^{\circ}\left(c 0.93, \mathrm{CHCl}_{3}\right) ;{ }^{1} \mathrm{H} \mathrm{NMR}\left(500 \mathrm{MHz} \mathrm{CDCl}_{3}\right) \delta 0.14(3 \mathrm{H}, \mathrm{s}), 0.18(3 \mathrm{H}, \mathrm{s}), 0.88(9 \mathrm{H}, \mathrm{s}), 1.35$ $(9 \mathrm{H}, \mathrm{s}), 1.41(1 \mathrm{H}, \mathrm{ddd}, J=3.6,8.4,12.2 \mathrm{~Hz}), 1.45(3 \mathrm{H}, \mathrm{s}), 1.91-2.00(1 \mathrm{H}, \mathrm{m}), 2.36-2.44(1 \mathrm{H}, \mathrm{m}), 2.53(1 \mathrm{H}$, dd, $J=10.3,13.3 \mathrm{~Hz}), 2.57-2.63(1 \mathrm{H}, \mathrm{m}), 2.72-2.77(1 \mathrm{H}, \mathrm{m}), 2.83(1 \mathrm{H}, \mathrm{dd}, J=5.2,13.3 \mathrm{~Hz}), 2.85-2.92(1 \mathrm{H}$, m), $3.51(3 \mathrm{H}, \mathrm{s}), 3.84(3 \mathrm{H}, \mathrm{s}), 3.95-3.97(1 \mathrm{H}, \mathrm{m}), 4.15(1 \mathrm{H}, \mathrm{dd}, J=2.4,7.3 \mathrm{~Hz}), 4.62(1 \mathrm{H}, \mathrm{d}, J=7.3 \mathrm{~Hz})$, 4.84-4.87 (1H, m), 4.93-4.98 (1H, m), 5.14-5.17 (1H, m), 5.19-5.24 (2H, s), $5.22(1 \mathrm{H}, \mathrm{m}), 5.88,(1 \mathrm{H}$, ddd, $J=7.8,10.2,17.8 \mathrm{~Hz}), 6.78-6.8 /(2 \mathrm{H}, \mathrm{m}), 7.00(1 \mathrm{H}, \mathrm{d}, J=1.3 \mathrm{~Hz}) ;{ }^{13} \mathrm{C} \mathrm{NMR}\left(125 \mathrm{MHz}, \mathrm{CDCl}_{3}\right) \delta-4.15$, $-3.57,18.0,24.2,25.8,26.4,36.2,38.2,42.0,43.0,44.8,49.2,55.9,56.1,70.0,78.5,78.8,95.6,108.6$, $111.6,111.7,114.1,117.0,122.2,134.6,145.8,146.1,146.3,147.8$; IR (neat) 2932, 2858, 1637, 1512, 1464, 1381, 1259, 1209, 1157, 1134, 1078, 1059, 1030, 1005, 908, 864, 835, 810, $775 \mathrm{~cm}^{-1}$; HREIMS m/z calcd for $\mathrm{C}_{31} \mathrm{H}_{48} \mathrm{O}_{6} \mathrm{Si}\left(\mathrm{M}^{+}\right), 544.3220$, found 544.3224.

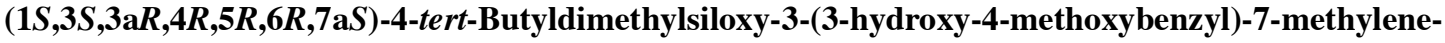
1-(vinyl)octahydroindene-5,6-diol (16).

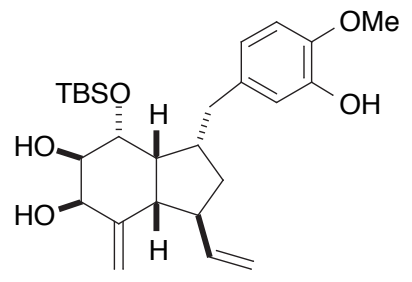

16

A solution of trifluoroacetic acid-water $(11 \mathrm{ml}, 10: 1)$ was added dropwise to a stirred solution of $(1 S, 3 S, 3 \mathrm{a} S, 5 R, 6 R, 7 R, 7 \mathrm{a} R)$-7-tert-butyldimethylsiloxy-5,6-O-isopropylidenedioxy-1-[4-methoxy-3(methoxymethoxy)benzyl]-4-methylene-3-(vinyl)octahydroindene (3) (177 $\mathrm{mg}, 0.33 \mathrm{mmol}$ ) in THF (1 $\mathrm{ml}$ ) at $0^{\circ} \mathrm{C}$. After $10 \mathrm{~min}$, the mixture was neutralized with $6 \mathrm{M}$ aqueous sodium hydroxide and then extracted with ethyl acetate $(2 \times 50 \mathrm{ml})$. The organic layer was washed with saturated aqueous sodium hydrogen carbonate $(2 \times 10 \mathrm{ml})$ and brine $(10 \mathrm{ml})$, then dried over $\mathrm{Na}_{2} \mathrm{SO}_{4}$. Concentration of the solvent in vacuo afforded a residue, which was purified by column chromatography (hexane-ethyl acetate, 3:1) to 
give $16(128 \mathrm{mg}, 86 \%)$ as a colorless oil: $[\alpha]_{\mathrm{D}}^{20}+19.2^{\circ}\left(c 1.97, \mathrm{CHCl}_{3}\right) ;{ }^{1} \mathrm{H} \mathrm{NMR}\left(500 \mathrm{MHz}, \mathrm{CDCl}_{3}\right) \delta$ $0.22(3 \mathrm{H}, \mathrm{s}), 0.26(3 \mathrm{H}, \mathrm{s}), 0.98(9 \mathrm{H}, \mathrm{s}), 1.59(1 \mathrm{H}, \mathrm{ddd}, J=5.7,10.0,15.8 \mathrm{~Hz}), 1.74(1 \mathrm{H}, \mathrm{d}, J=6.1 \mathrm{~Hz}), 1.93$ $(1 \mathrm{H}, \mathrm{d}, J=7.5 \mathrm{~Hz}), 1.94-2.02(1 \mathrm{H}, \mathrm{m}), 2.27-2.32(1 \mathrm{H}, \mathrm{m}), 2.41-2.51(2 \mathrm{H}, \mathrm{m}), 2.69(1 \mathrm{H}, \mathrm{dd}, J=8.9,13.6$ $\mathrm{Hz}), 2.77-2.86(2 \mathrm{H}, \mathrm{m}), 3.86(3 \mathrm{H}, \mathrm{s}), 3.95-3.99(1 \mathrm{H}, \mathrm{m}), 4.26(1 \mathrm{H}, \mathrm{t}, J=4.0 \mathrm{~Hz}), 4.70-4.74(1 \mathrm{H}, \mathrm{m}), 4.80-$ $4.86(1 \mathrm{H}, \mathrm{m}), 4.86-4.90(1 \mathrm{H}, \mathrm{m}), 4.95(1 \mathrm{H}, \mathrm{s}), 5.15(1 \mathrm{H}, \mathrm{t}, J=1.8 \mathrm{~Hz}), 5.54(1 \mathrm{H}, \mathrm{s}), 5.62(1 \mathrm{H}, \mathrm{ddd}, J=8.3$, 10.1, $18.3 \mathrm{~Hz}), 6.66(1 \mathrm{H}, \mathrm{dd}, J=2.1,8.2 \mathrm{~Hz}), 6.76(1 \mathrm{H}, \mathrm{d}, J=8.2 \mathrm{~Hz}), 6.78(1 \mathrm{H}, \mathrm{d}, J=2.1 \mathrm{~Hz}) ;{ }^{13} \mathrm{C}$ NMR $\left(125 \mathrm{MHz}, \mathrm{CDCl}_{3}\right) \delta-4.05,-3.37,18.1,26.1,35.5,37.1,43.2,44.2,47.5,54.3,56.0,68.2,72.7,75.4$, 110.6, 110.8, 113.4, 114.6, 119.6, 135.3, 142.8, 144.7, 145.4, 145.8; IR (neat) 3422, 2932, 2858, 1639, 1591, 1512, 1442, 1259, 1130, 1062, 1037, 904, 873, 835, 775, $706 \mathrm{~cm}^{-1}$; HRFABMS m/z calcd for $\mathrm{C}_{26} \mathrm{H}_{41} \mathrm{O}_{5} \mathrm{Si}\left[(\mathrm{M}+\mathrm{H})^{+}\right], 461.2723$, found 461.2697 .

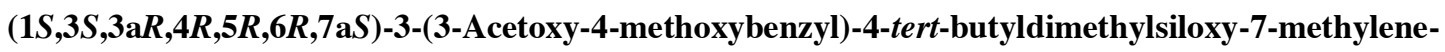
1-(vinyl)octahydroindene-5,6-diol (17).

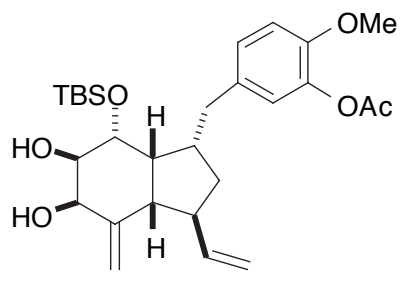

17

$2 \mathrm{M}$ aqueous sodium hydroxide $(0.38 \mathrm{ml})$ and acetic anhydride $(76 \mu 1,0.81 \mathrm{mmol})$ were added dropwise to a stirred solution of $(1 S, 3 S, 3 \mathrm{a} R, 4 R, 5 R, 6 R, 7 \mathrm{a} S)$-4-tert-butyldimethylsiloxy-3-(3-hydroxy-4methoxybenzyl)-7-methylene-1-(vinyl)octahydroindene-5,6-diol (16) (128 mg, $0.28 \mathrm{mmol}$ ) in 2-propanol $(1.7 \mathrm{ml})$ at room temperature. After $30 \mathrm{~min}$, the mixture was diluted with ethyl acetate $(30 \mathrm{ml})$. The organic layer was washed with water $(10 \mathrm{ml})$ and brine $(10 \mathrm{ml})$, then dried over $\mathrm{Na}_{2} \mathrm{SO}_{4}$. Concentration of the solvent in vacuo gave a residue, which was purified by column chromatography (benzene-ethyl acetate, 10:1) to give $17(127 \mathrm{mg}, 91 \%)$ as a colorless oil: $[\alpha]_{\mathrm{D}}{ }^{20}+20.9^{\circ}\left(c 0.80, \mathrm{CHCl}_{3}\right) ;{ }^{1} \mathrm{H}$ NMR (500 $\left.\mathrm{MHz}, \mathrm{CDCl}_{3}\right) \delta 0.21$ (3H. s), $0.24(3 \mathrm{H}, \mathrm{s}), 0.97(9 \mathrm{H}, \mathrm{s}), 1.56-1.63(1 \mathrm{H}, \mathrm{m}), 1.78(1 \mathrm{H}, \mathrm{d}, J=5.8 \mathrm{~Hz}), 1.92$ $(1 \mathrm{H}, \mathrm{d}, J=7.6 \mathrm{~Hz}), 1.94-2.01(1 \mathrm{H}, \mathrm{m}), 2.27-2.30(1 \mathrm{H}, \mathrm{m}), 2.31(3 \mathrm{H}, \mathrm{s}), 2.39-2.49(2 \mathrm{H} . \mathrm{m}), 2.72(1 \mathrm{H}, \mathrm{dd}$, $J=8.8,13.7 \mathrm{~Hz}), 2.77-2.86(1 \mathrm{H}, \mathrm{dd}, J=6.3,13.7 \mathrm{~Hz}), 2.83(1 \mathrm{H}, \mathrm{m}), 3.80(3 \mathrm{H}, \mathrm{s}), 3.95-3.99(1 \mathrm{H}, \mathrm{m}), 4.23-$ $4.26(1 \mathrm{H}, \mathrm{m}), 4.68-4.72(1 \mathrm{H}, \mathrm{m}), 4.80-4.85(1 \mathrm{H}, \mathrm{m}), 4.88(1 \mathrm{H}, \mathrm{dd}, J=1.3,10.0 \mathrm{~Hz}), 4.94(1 \mathrm{H}, \mathrm{s}), 5.14-$ $5.16(1 \mathrm{H}, \mathrm{m}), 5.62(1 \mathrm{H}, \mathrm{ddd}, J=8.3,10.0,17.1 \mathrm{~Hz}), 6.85(1 \mathrm{H}, \mathrm{d}, J=2.1 \mathrm{~Hz}), 6.87(1 \mathrm{H}, \mathrm{d}, J=8.3 \mathrm{~Hz}), 7.00$ $(1 \mathrm{H}, \mathrm{dd}, J=2.1,8.3 \mathrm{~Hz}) ;{ }^{13} \mathrm{C}$ NMR $\left(125 \mathrm{MHz}, \mathrm{CDCl}_{3}\right) \delta-4.06,-3.36,18.1,20.7,26.1,35.3,37.1,43.1$, 44.2, 47.5, 54.3, 55.9, 68.2, 72.7, 75.4, 110.9, 112.4, 113.5, 122.6, 126.4, 134.6, 139.5, 142.7, 145.7, 149.1, 168.3; IR (neat) 3449, 2955, 2932, 2858, 1768, 1639, 1512, 1464, 1442, 1369, 1263, 1205, 1122, 1062, 1035, 902, 873, 835, 775, $706 \mathrm{~cm}^{-1}$; HRFABMS m/z calcd for $\mathrm{C}_{28} \mathrm{H}_{43} \mathrm{O}{ }_{6} \mathrm{Si}\left[(\mathrm{M}+\mathrm{H})^{+}\right], 503.2829$, found 503.2832 . 


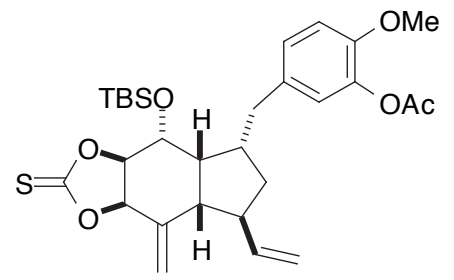

18

A solution of thiophosgene $(18 \mu \mathrm{l}, 0.24 \mathrm{mmol})$ in dry dichloromethane $(0.5 \mathrm{ml})$ was added dropwise to a stirred mixture of $(1 S, 3 S, 3 \mathrm{a} R, 4 R, 5 R, 6 R, 7 \mathrm{a} S)$-3-(3-acetoxy-4-methoxybenzyl)-4-tert-butyldimethylsiloxy7-methylene-1-(vinyl)octahydroindene-5,6-diol (17) $(60.0 \mathrm{mg}, 0.12 \mathrm{mmol})$ in dry dichloromethane $(2 \mathrm{ml})$ containing 4-dimethylaminopyridine $(72.0 \mathrm{mg}, 0.60 \mathrm{mmol})$ at room temperature. After $30 \mathrm{~min}$, silica gel $(1.0 \mathrm{~g})$ was added to the reaction mixture, and the solvent was carefully evaporated off in vacuo. The resulting solid was charged on the top of a silica gel column chromatography, and elution using hexaneethyl acetate (10:1) gave $18(60 \mathrm{mg}, 93 \%)$ as a colorless oil: $[\alpha]_{\mathrm{D}}{ }^{20}+25.3^{\circ}\left(c 0.60, \mathrm{CHCl}_{3}\right) ;{ }^{1} \mathrm{H} \mathrm{NMR}(500$ $\left.\mathrm{MHz}, \mathrm{CDCl}_{3}\right) \delta 0.16(3 \mathrm{H}, \mathrm{s}), 0.22(3 \mathrm{H}, \mathrm{s}), 0.87(9 \mathrm{H}, \mathrm{s}), 1.44-1.50(1 \mathrm{H}, \mathrm{m}), 1.88-1.96(1 \mathrm{H}, \mathrm{m}), 2.31(3 \mathrm{H}$, s), 2.31-2.34 $(1 \mathrm{H}, \mathrm{m}), 2.40-2.52(2 \mathrm{H}, \mathrm{m}), 2.79-2.84(1 \mathrm{H}, \mathrm{m}), 2.86-2.93(2 \mathrm{H}, \mathrm{m}), 3.80(3 \mathrm{H}, \mathrm{s}), 4.15-4.18$ $(1 \mathrm{H}, \mathrm{m}), 4.85(1 \mathrm{H}, \mathrm{dd}, J=2.4,8.5 \mathrm{~Hz}), 4.91,(1 \mathrm{H}, \mathrm{d}, J=10.1 \mathrm{~Hz}), 4.94-4.99(1 \mathrm{H}, \mathrm{m}), 5.28-5.33(2 \mathrm{H}, \mathrm{m})$, $5.47(1 \mathrm{H}, \mathrm{d}, J=2.5 \mathrm{~Hz}), 5.85(1 \mathrm{H}, \mathrm{ddd}, J=7.8,10.1,17.2 \mathrm{~Hz}), 6.82(1 \mathrm{H}, \mathrm{d}, J=2.1 \mathrm{~Hz}), 6.89(1 \mathrm{H}, \mathrm{d}, J=8.3$ $\mathrm{Hz}), 6.97(1 \mathrm{H}, \mathrm{dd}, J=2.1,8.3 \mathrm{~Hz}) ;{ }^{13} \mathrm{C} \mathrm{NMR}\left(125 \mathrm{MHz}, \mathrm{CDCl}_{3}\right) \delta-4.41,-3.49,17.9,20.7,25.6,35.7$, 38.0, 42.5, 43.0, 43.9, 49.6, 55.9, 67.6, 82.1, 85.3, 112.5, 112.6, 118.8, 122.7, 126.5, 133.5, 139.6, 141.1, 144.6, 149.4, 169.0, 190.8; IR (neat) 3076, 2955, 2932, 2858, 1768, 1639, 1583, 1512, 1464, 1442, 1369, 1331, 1267, 1205, 1163, 1124, 1089, 1064, 1032, 995, 958, 904, 866, 837, 777, $758 \mathrm{~cm}^{-1}$; HREIMS m/z calcd for $\mathrm{C}_{29} \mathrm{H}_{40} \mathrm{O}_{6} \mathrm{SSi}\left(\mathrm{M}^{+}\right), 544.2315$, found 544.2316.

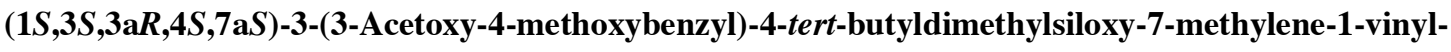
1,2,3,3a,7,7a-hexahydroindene (19).

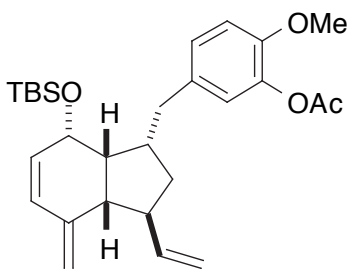

19

A solution of $(3 \mathrm{a} R, 4 R, 4 \mathrm{a} R, 5 S, 7 S, 7 \mathrm{a} S)$-5-(3-acetoxy-4-methoxybenzyl)-4-tert-butyldimethylsiloxy-8methylene-7-(vinyl)octahydroindeno[5,6- $d][1,3]$ dioxole-2-thione (18) (24.0 $\mathrm{mg}, 44 \mu \mathrm{mol})$ in triethyl phosphite $(4.4 \mathrm{ml})$ was heated at reflux for $2 \mathrm{~h}$ under argon. After cooling, excess triethyl phosphite was removed using a short column chromatography (hexane). The combined fractions were concentrated in 
vacuo to afford a residue, which was purified by column chromatography (benzene-ethyl acetate, 100:1) to give (19) $(16 \mathrm{mg}, 78 \%)$ as a colorless oil: $[\alpha]_{\mathrm{D}}{ }^{20}+98.4^{\circ}\left(c 1.07, \mathrm{CHCl}_{3}\right) ;{ }^{1} \mathrm{H} \mathrm{NMR}\left(500 \mathrm{MHz}, \mathrm{CDCl}_{3}\right)$

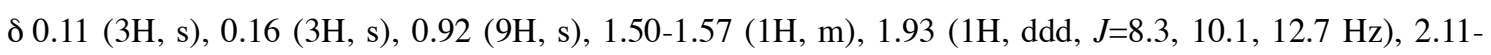
$2.16(1 \mathrm{H}, \mathrm{m}), 2.30(2 \mathrm{H}, \mathrm{s}), 2.38-2.50(2 \mathrm{H}, \mathrm{m}), 2.70(1 \mathrm{H}, \mathrm{dd}, J=9.3,13.7 \mathrm{~Hz}), 2.78-2.87(1 \mathrm{H}, \mathrm{m}), 2.96(1 \mathrm{H}$, dd, $J=6.0,13.7 \mathrm{~Hz}), 3.80(3 \mathrm{H}, \mathrm{s}), 4.45(1 \mathrm{H}, \mathrm{t}, J=4.9 \mathrm{~Hz}), 4.84-4.92(3 \mathrm{H}, \mathrm{m}), 4.95(1 \mathrm{H}, \mathrm{s}), 5.73(1 \mathrm{H}, \mathrm{ddd}$, $J=8.8,10.0,16.9 \mathrm{~Hz}), 5.83(1 \mathrm{H}, \mathrm{dd}, J=4.9,9.7 \mathrm{~Hz}), 6.13(1 \mathrm{H}, \mathrm{d}, J=9.8 \mathrm{~Hz}), 6.85-6.89(2 \mathrm{H}, \mathrm{m}), 7.01(1 \mathrm{H}$, dd, $J=2.0,8.3 \mathrm{~Hz}) ;{ }^{13} \mathrm{C}$ NMR $\left(125 \mathrm{MHz}, \mathrm{CDCl}_{3}\right) \delta-4.08,-3.29,18.1,20.7,26.0,35.7,43.9,45.9,47.9$, 49.6, 55.9, 65.5, 112.2, 113.5, 114.3, 122.8, 126.6, 129.9, 131.1, 135.4, 139.5, 143.3, 144.0, 148.9, 169.0; IR (neat) 2928.2, 2856.8, 1770.8, 1512.3, 1464.1, 1367.6, 1261.5, 1203.6, 1124.6, 1030.0, 881.5, 835.2, $773.5 \mathrm{~cm}^{-1}$; HREIMS m/z calcd for $\mathrm{C}_{28} \mathrm{H}_{40} \mathrm{O}_{4} \mathrm{Si}\left(\mathrm{M}^{+}\right), 468.2696$, found 468.2700 .

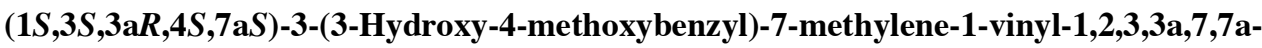

\section{hexahydroindene-4-ol (20).}

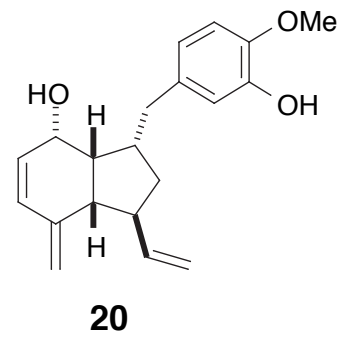

Tetrabutylammonium fluoride (TBAF) in THF ( $1 \mathrm{M}, 0.43 \mathrm{ml}, 0.43 \mathrm{mmol}$ ) was added in a stirred solution of $\quad(1 S, 3 S, 3 \mathrm{a} R, 4 S, 7 \mathrm{a} S)$-3-(3-acetoxy-4-methoxybenzyl)-4-tert-butyldimethylsiloxy-7-methylene-1-vinyl1,2,3,3a,7,7a-hexahydroindene (19) $(20.0 \mathrm{mg}, 43 \mu \mathrm{mol})$ in THF $(1 \mathrm{ml})$ at room temperature. After $48 \mathrm{~h}$, the reaction was quenched with saturated aqueous ammonium chloride $(0.5 \mathrm{ml})$, and extracted with ethyl acetate $(2 \times 15 \mathrm{ml})$. The combined extracts were washed with saturated aqueous sodium hydrogen carbonate $(2 \times 5 \mathrm{ml})$ and brine $(5 \mathrm{ml})$, then dried over $\mathrm{Na}_{2} \mathrm{SO}_{4}$. Concentration of the solvent in vacuo afforded a residue, which was purified by column chromatography (benzene-ethyl acetate, 4:1) to give 20 $(11 \mathrm{mg}, 83 \%)$ as a colorless oil: $[\alpha]_{\mathrm{D}}{ }^{20}+74.6^{\circ}\left(c 0.35, \mathrm{CHCl}_{3}\right) ;{ }^{1} \mathrm{H} \mathrm{NMR}\left(500 \mathrm{MHz}, \mathrm{CDCl}_{3}\right) \delta 1.05(1 \mathrm{H}$, br), $1.65(1 \mathrm{H}$, ddd, $J=6.0,10.1,16.1 \mathrm{~Hz}), 1.98-2.03(1 \mathrm{H}, \mathrm{m}), 2.04-2.10(1 \mathrm{H}, \mathrm{m}), 2.44(1 \mathrm{H}, \mathrm{dd}, J=6.3,10.8$ $\mathrm{Hz}), 2.47-2.55(1 \mathrm{H}, \mathrm{m}), 2.55-2.61(1 \mathrm{H}, \mathrm{m}), 2.87(1 \mathrm{H}, \mathrm{dd}, J=8.3,13.7 \mathrm{~Hz}), 2.98(1 \mathrm{H}, \mathrm{dd}, J=7.2,13.7 \mathrm{~Hz})$, $3.86(3 \mathrm{H}, \mathrm{s}), 4.36(1 \mathrm{H}, \mathrm{br}), 4.82-4.88(2 \mathrm{H}, \mathrm{m}), 4.90(1 \mathrm{H}, \mathrm{dd}, J=1.9,10.0 \mathrm{~Hz}), 5.01(1 \mathrm{H}, \mathrm{s}), 5.54(1 \mathrm{H}, \mathrm{br})$, $5.70(1 \mathrm{H}, \mathrm{ddd}, J=8.8,10.0,18.7 \mathrm{~Hz}), 5.93(1 \mathrm{H}, \mathrm{dd}, J=5.6,9.7 \mathrm{~Hz}), 6.19(1 \mathrm{H}, \mathrm{d}, J=9.7 \mathrm{~Hz}), 6.73(1 \mathrm{H}, \mathrm{dd}$, $J=1.9,8.2 \mathrm{~Hz}), 6.76(1 \mathrm{H}, \mathrm{d}, J=8.2 \mathrm{~Hz}), 6.84(1 \mathrm{H}, \mathrm{d}, J=1.9 \mathrm{~Hz}) ;{ }^{13} \mathrm{C} \mathrm{NMR}\left(125 \mathrm{MHz}, \mathrm{CDCl}_{3}\right) \delta 36.7,37.9$, 44.4, 44.9, 48.6, 49.9, 55.9, 64.3, 110.4, 113.9, 114.7, 115.8, 119.9, 128.1, 131.5, 135.8, 142.0, 143.7, 144.5, 145.3; IR (neat) 3510, 2924, 1591, 1512, 1442, 1273, 1130, 1008, 904, 80, $761 \mathrm{~cm}^{-1}$; HREIMS m/z calcd for $\mathrm{C}_{20} \mathrm{H}_{24} \mathrm{O}_{3}\left(\mathrm{M}^{+}\right), 312.1725$, found 312.1729 . 
(1S,3S,3aR,4S,7aS)-3-(3-Dichloroacetoxy-4-methoxybenzyl)-1,2,3,3a,7,7a-hexahydro-7-methylene-1(vinyl)indene-4-ol (21).

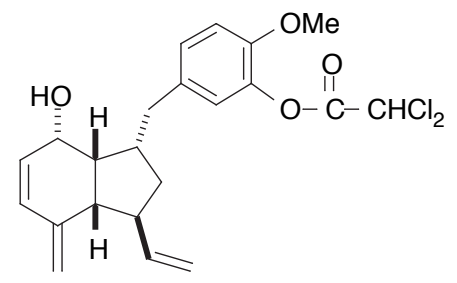

21

A solution of dichloroacetic anhydride $(10 \mu \mathrm{l}, 65 \mu \mathrm{mol})$ in dry dichloromethane $(0.2 \mathrm{ml})$ was added very slowly to a stirred solution of $(1 S, 3 S, 3 \mathrm{a} R, 4 S, 7 \mathrm{a} S)$-3-(3-hydroxy-4-methoxybenzyl)-7-methylene-1-vinyl1,2,3,3a,7,7a-hexahydroindene-4-ol (20) $(17.0 \mathrm{mg}, 54 \mu \mathrm{mol})$ in dry dichloromethane (1 ml) containing pyridine $(9.0 \mu \mathrm{l}, 0.1 \mathrm{mmol})$ at room temperature. The reaction mixture was diluted with ethyl acetate (10 $\mathrm{ml}$ ), and the organic layer was washed with brine $(5 \mathrm{ml})$, and dried over $\mathrm{Na}_{2} \mathrm{SO}_{4}$. Concentration of the solvent in vасио afforded a residue, which was purified by column chromatography (benzene-ethyl acetate, 20:1 ) to give $21(12 \mathrm{mg}, 52 \%)$ as a white cloudy oil: $[\alpha]_{\mathrm{D}}{ }^{20}+71.5^{\circ}\left(c 0.37, \mathrm{CHCl}_{3}\right) ;{ }^{1} \mathrm{H}$ NMR $\left(500 \mathrm{MHz}, \mathrm{CDCl}_{3}\right) \delta 1.02(1 \mathrm{H}, \mathrm{d}, J=6.3 \mathrm{~Hz}), 1.64(1 \mathrm{H}, \mathrm{ddd}, J=6.0,10.1,19.0 \mathrm{~Hz}), 1.97-2.03(1 \mathrm{H}, \mathrm{m})$, 2.05-2.10 (1H, m), 2.43-2.47 (1H, m), 2.47-2.54 (1H, m), 2.54-2.61 (1H, m), 2.93 (1H, dd, J=8.6, 13.9 Hz), 3.03 (1H, dd, J=6.9, $13.9 \mathrm{~Hz}), 4.31-4.36(1 \mathrm{H}, \mathrm{m}), 4.83-4.88(2 \mathrm{H}, \mathrm{m}), 4.92(1 \mathrm{H}, \mathrm{dd}, J=1.8,9.9 \mathrm{~Hz})$, $5.02(1 \mathrm{H}, \mathrm{s}), 5.70(1 \mathrm{H}, \mathrm{ddd}, J=8.8,9.9,18.7 \mathrm{~Hz}), 5.91-5.96(1 \mathrm{H}, \mathrm{m}), 6.18(1 \mathrm{H}, \mathrm{s}), 6.20(1 \mathrm{H}, \mathrm{d}, J=9.6 \mathrm{~Hz})$, $6.91(1 \mathrm{H}, \mathrm{d}, J=8.4 \mathrm{~Hz}), 7.01(1 \mathrm{H}, \mathrm{d}, J=2.0 \mathrm{~Hz}), 7.14(1 \mathrm{H}, \mathrm{dd}, J=2.0,8.4 \mathrm{~Hz}) ;{ }^{13} \mathrm{C} \mathrm{NMR}(125 \mathrm{MHz}$, $\left.\mathrm{CDCl}_{3}\right) \delta 35.9,37.8,44.3,44.9,48.6,49.9,56.1,64.0,64.3,112.6,114.0,116.0,121.9,127.6,128.0$, 131.6, 135.4, 138.8, 141.9, 143.5, 148.6, 162.5; IR (neat) 3568, 2926, 2345, 1782, 1512, 1269, 1141, $1028,815,407 \mathrm{~cm}^{-1}$; HREIMS m/z calcd for $\mathrm{C}_{22} \mathrm{H}_{24} \mathrm{Cl}_{2} \mathrm{O}_{4}\left(\mathrm{M}^{+}\right), 422.1052$, found 422.1050 .

(1S,3S,3aS,7aS)-3-(3-Dichloroacetyl-4-methoxybenzyl)-1,2,3,3a,7,7a-hexahydro-7-methylene-1(vinyl)indene-4-one (22).

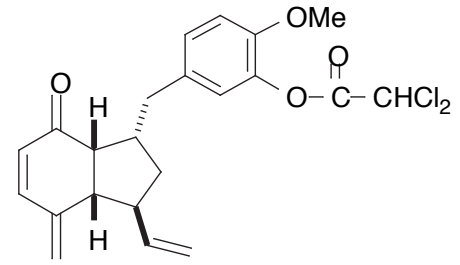

22

Dess-Martin periodinane $(34.0 \mathrm{mg}, 81 \mu \mathrm{mol})$ was added to a stirred solution of $(1 S, 3 S, 3 \mathrm{a} R, 4 S, 7 \mathrm{a} S)-3-(3-$ dichloroacetoxy-4-methoxybenzyl)-1,2,3,3a,7,7a-hexahydro-7-methylene-1-(vinyl)indene-4-ol (21) (17.0 $\mathrm{mg}, 40 \mu \mathrm{mol})$ in dry dichloromethane $(1 \mathrm{ml})$ at room temperature. After $15 \mathrm{~min}$, the reaction was quenched with $20 \%$ aqueous sodium thiosulfate $(0.4 \mathrm{ml})$, and the mixture was extracted with ethyl acetate $(2 \times 10 \mathrm{ml})$. The combined extracts were washed with brine $(5 \mathrm{ml})$, then dried over $\mathrm{Na}_{2} \mathrm{SO}_{4}$. Concentration of the solvent in vacuo afforded a residue, which was purified by column chromatography (hexane-ethyl acetate, $4: 1)$ to give $22(16 \mathrm{mg}, 95 \%)$ as a white cloudy oil: $[\alpha]_{\mathrm{D}}^{20}+22.4^{\circ}\left(c 0.83, \mathrm{CHCl}_{3}\right)$; 
${ }^{1} \mathrm{H}$ NMR $\left(500 \mathrm{MHz}, \mathrm{CDCl}_{3}\right) \delta$ 1.58-1.64 (1H, m), 1.69-1.77 $(1 \mathrm{H}, \mathrm{m}), 2.42-2.51(1 \mathrm{H}, \mathrm{m}), 2.54-2.63(2 \mathrm{H}$, m), 2.73-2.78 (1H, m), 2.78-2.82 (1H, m), $3.16(1 \mathrm{H}, \mathrm{dd}, J=6.9,13.6 \mathrm{~Hz}), 3.80(3 \mathrm{H}, \mathrm{s}), 4.90-4.95(1 \mathrm{H}, \mathrm{m})$, 4.96-4.99 (1H, m), $5.29(1 \mathrm{H}, \mathrm{d}, J=1.1 \mathrm{~Hz}), 5.32(1 \mathrm{H}, \mathrm{s}), 5.75(1 \mathrm{H}$, ddd, $J=8.2,10.1,18.3 \mathrm{~Hz}), 5.92(1 \mathrm{H}$, dd, J=0.6, $9.9 \mathrm{~Hz}), 6.18(1 \mathrm{H}, \mathrm{s}), 6.89(1 \mathrm{H}, \mathrm{d}, J=8.4 \mathrm{~Hz}), 6.97(1 \mathrm{H}, \mathrm{d}, J=8.4 \mathrm{~Hz}), 6.98(1 \mathrm{H}, \mathrm{d}, J=2.1 \mathrm{~Hz})$, $7.11(1 \mathrm{H}, \mathrm{dd}, J=2.1,8.4 \mathrm{~Hz}) ;{ }^{13} \mathrm{C} \mathrm{NMR}\left(125 \mathrm{MHz}, \mathrm{CDCl}_{3}\right) \delta 35.5,35.6,36.3,48.7,49.1,51.0$, 56.1, 64.0, 112.6, 114.5, 120.1, 122.2, 128.0, 128.3, 134.8, 138.7, 141.6, 142.5, 145.8, 148.8, 162.5, 200.2; IR (neat) 2932, 1784, 1660, 1512, 1442, 1267, 1217, 1141, 1028, 912, $814 \mathrm{~cm}^{-1}$; HREIMS m/z calcd for $\mathrm{C}_{22} \mathrm{H}_{22} \mathrm{Cl}_{2} \mathrm{O}_{4}\left(\mathrm{M}^{+}\right), 420.0895$, found 420.0888 .

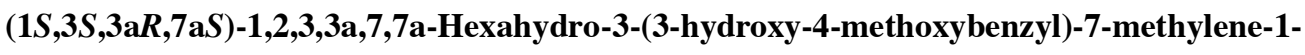
(vinyl)indene-4-one (1) (ottelione A).

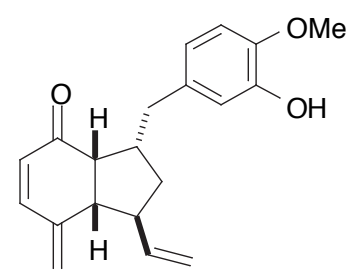

1 (ottelione A )

(1S,3S,3aS,7aS)-3-(3-Dichloroacetyl-4-methoxybenzyl)-1,2,3,3a,7,7a-hexahydro-7-methylene-1-

(vinyl)indene-4-one (22) (13.0 mg, 31 umol) was dissolved in a solution of $50 \%$ aqueous sodium hydrogen carbonate-acetonitrile $(1.0 \mathrm{ml}, 1: 1)$, and the mixture was stirred for $1 \mathrm{~h}$ at room temperature. The mixture was diluted with ethyl acetate $(10 \mathrm{ml})$, and the organic layer was washed with brine $(5 \mathrm{ml})$, then dried over $\mathrm{Na}_{2} \mathrm{SO}_{4}$. Concentration of the solvent in vacuo afforded a residue, which was purified by column chromatography (benzene-ethyl acetate, $20: 1)$ to give $\mathbf{1}(8.5 \mathrm{mg}, 90 \%)$ as white cloudy oil: $[\alpha]_{\mathrm{D}}{ }^{25}$ $+17.3^{\circ}\left(c\right.$ 0.55, $\left.\mathrm{CHCl}_{3}\right)$; ${ }^{1} \mathrm{H}$ NMR $\left(500 \mathrm{MHz}, \mathrm{CDCl}_{3}\right) \delta 1.52-1.59(1 \mathrm{H}, \mathrm{m}), 1.69-1.76(1 \mathrm{H}, \mathrm{m}), 2.46-2.55$ $(2 \mathrm{H}, \mathrm{m}), 2.58-2.66(1 \mathrm{H}, \mathrm{m}), 2.78(1 \mathrm{H}, \mathrm{t}, J=8.2 \mathrm{~Hz}), 2.86(1 \mathrm{H}, \mathrm{dd}, \mathrm{J}=5.9,8.2 \mathrm{~Hz}), 3.01-3.08(1 \mathrm{H}, \mathrm{m}), 3.85$ $(3 \mathrm{H}, \mathrm{s}), 4.91-4.99(2 \mathrm{H}, \mathrm{m}), 5.30-5.34(2 \mathrm{H}, \mathrm{m}), 5.53(1 \mathrm{H}, \mathrm{br}), 5.76(1 \mathrm{H}, \mathrm{ddd}, J=8.2,10.1,18.2 \mathrm{~Hz}), 5.39$ $(1 \mathrm{H}, \mathrm{d}, J=10.0 \mathrm{~Hz}), 6.68(1 \mathrm{H}, \mathrm{dd}, J=2.0,8.2 \mathrm{~Hz}), 6.75(1 \mathrm{H}, \mathrm{d}, J=8.2 \mathrm{~Hz}), 6.79(1 \mathrm{H}, \mathrm{d}, J=2.0 \mathrm{~Hz}), 6.99$ $(1 \mathrm{H}, \mathrm{d}, J=9.9 \mathrm{~Hz}) ;{ }^{13} \mathrm{C} \mathrm{NMR}\left(125 \mathrm{MHz}, \mathrm{CDCl}_{3}\right) \delta 35.6,35.8,46.3,48.5,49.0,51.4,55.9,110.4,114.4$, $114.9,119.9,120.2,128.3,135.1,141.8,142.9,144.6,145.2,145.9,200.4$; IR (neat) 3439, 2926, 1658, 1589, 1510, 1440, 1275, 1236, 1130, 1030, 958, 800, $760 \mathrm{~cm}^{-1}$; HREIMS m/z calcd for $\mathrm{C}_{20} \mathrm{H}_{22} \mathrm{O}_{3}\left(\mathrm{M}^{+}\right)$, 310.1569 , found 310.1571 . 
(1S,3S,3aS,7aS)-1,2,3,3a,7,7a-Hexahydro-3-(3-hydroxy-4-methoxybenzyl)-7-methylene-1(vinyl)indene-4-one (2) (ottelione B).

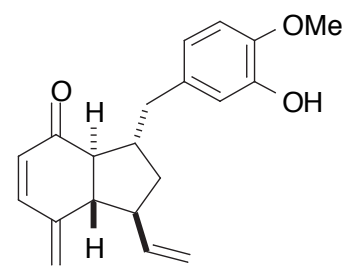

\section{2 (ottelione B )}

Potassium tert-butoxide $(8.0 \mathrm{mg}, 0.13 \mathrm{mmol})$ was added to a stirred solution of ottelione A (1) $(8.0 \mathrm{mg}$, $26 \mu \mathrm{mol})$ in tert-butyl alcohol $(1 \mathrm{ml})$ at room temperature. After $2 \mathrm{~h}$, the mixture was diluted with ethyl acetate $(10 \mathrm{ml})$. The organic layer was washed with saturated aqueous ammonium chloride $(2 \times 2 \mathrm{ml})$, saturated sodium hydrogen carbonate $(2 \times 2 \mathrm{ml})$ and brine $(2 \mathrm{ml})$, then dried over $\mathrm{Na}_{2} \mathrm{SO}_{4}$. Concentration of the solvent in vасиo afforded a residue, which was purified by column chromatography (hexane-ethyl acetate, 4:1) to give a mixture of $\mathbf{2}$ and $\mathbf{1}\left(6.3 \mathrm{mg}, 79 \%, \mathbf{2 : 1}=77: 23\right.$ determined by $500 \mathrm{MHz}{ }^{1} \mathrm{H}$ NMR) as colorless viscous oil. Isolation of $\mathbf{2}$ from this mixture was performed by means of HPLC (DAICEL CHIRALPAK $^{\circledR}$ AD-H, i.d. 4.6 x $250 \mathrm{~mm}$, hexane:2-propanol=4:1, $0.5 \mathrm{ml} / \mathrm{min}$; measurement of UV 254 nm sbsorbance, ${ }^{\mathrm{t}} \mathrm{R}-2$ : $34.7 \mathrm{~min},{ }^{\mathrm{t}} \mathrm{R}-\mathbf{1}$ : $\left.29.6 \mathrm{~min}\right)$ to give $2(1.8 \mathrm{mg}, 23 \%)$ as a white solid, $\mathrm{mp} 142.2-$ $143.0^{\circ} \mathrm{C} ;[\alpha]_{\mathrm{D}}{ }^{25}-330.0^{\circ}\left(c 0.18, \mathrm{CHCl}_{3}\right) ;{ }^{1} \mathrm{H}$ NMR $\left(500 \mathrm{MHz}, \mathrm{CDCl}_{3}\right) \delta 1.59(1 \mathrm{H}, \mathrm{ddd}, J=8.2,10.2,13.8$ $\mathrm{Hz}), 1.78(1 \mathrm{H}$, ddd, $J=6.7,9.8,13.8 \mathrm{~Hz}), 2.27(1 \mathrm{H}, \mathrm{dd}, J=9.8,13.9 \mathrm{~Hz}), 2.34(1 \mathrm{H}, \mathrm{dd}, J=10.2,13.9 \mathrm{~Hz})$, 2.46-2.56 (2H, m), 2.69-2.77 (1H, m), $3.14(1 \mathrm{H}, \mathrm{dd}, J=3.6,13.4 \mathrm{~Hz}), 3.86(3 \mathrm{H}, \mathrm{s}), 4.97-5.01(1 \mathrm{H}, \mathrm{m})$, 5.06-5.11 $(1 \mathrm{H}, \mathrm{m}), 5.30(1 \mathrm{H}, \mathrm{s}), 5.44(1 \mathrm{H}, \mathrm{s}), 5.52(1 \mathrm{H}, \mathrm{br}), 5.74(1 \mathrm{H}, \mathrm{ddd}, J=8.0,10.2,18.2 \mathrm{~Hz}), 5.94$ $(1 \mathrm{H}, \mathrm{d}, J=9.7 \mathrm{~Hz}), 6.69(1 \mathrm{H}, \mathrm{dd}, J=2.0,8.1 \mathrm{~Hz}), 6.76(1 \mathrm{H}, \mathrm{d}, J=8.1 \mathrm{~Hz}), 6.80(1 \mathrm{H}, \mathrm{d}, J=2.0 \mathrm{~Hz}), 7.00(1 \mathrm{H}$, d, $J=9.7 \mathrm{~Hz}) ;{ }^{13} \mathrm{C}$ NMR $\left(125 \mathrm{MHz}, \mathrm{CDCl}_{3}\right) \delta 36.9,37.9,40.6,44.5,50.5,55.9,58.3,110.5,114.6,115.3$, 117.1, 120.5, 128.7, 134.1, 141.5, 144.8, 144.9, 125.2, 147.6, 200.6; IR (KBr) 3395, 2930, 1664, 1589, $1512,1440,1275,1176,1126,1035,916,808,761 \mathrm{~cm}^{-1}$; HRFABMS m/z calcd for $\mathrm{C}_{20} \mathrm{H}_{23} \mathrm{O}_{3}\left[(\mathrm{M}+\mathrm{H})^{+}\right]$, 311.1647, found 311.1633 . 


\section{Reference}

1. 4-Bromo-1-methoxy-2-(methoxymethoxy)benzene (9) was prepared starting from the known 5bromo-2-methoxyphenol [ A. Diaz, J. G. Siro, J. L. Garcia-Navio, J. J. Vaquero, J. Alvarez-Builla, Synthesis 1997, 559 ] as follows.<smiles>COc1ccc(Br)cc1O</smiles><smiles>CCN[P+](=O)C(OC)OC</smiles>

Diisopropylethylamine $(10.6 \mathrm{ml}, 62 \mathrm{mmol})$ and chloromethylmethylether $(3.77 \mathrm{ml}, 49 \mathrm{mmol})$ were added successively to a stirred solution of 5-bromo-2-methoxyphenol (8.40 g, $41 \mathrm{mmol})$ in dichloromethane $(40 \mathrm{ml})$ at $0^{\circ} \mathrm{C}$ under argon, and the mixture was gradually warmed up to room temperature. After $4 \mathrm{~h}$, the reaction was quenched with saturated ammonium chloride (40 ml), and the resulting mixture was extracted with ether $(2 \mathrm{x} 400 \mathrm{ml})$. The combined extracts were washed successively with $3 \% \mathrm{HCl}(2 \times 100 \mathrm{ml})$, saturated aqueous sodium hydrogen carbonate $(2 \times 100 \mathrm{ml})$, and brine $(2 \times 100 \mathrm{ml})$, then dried over $\mathrm{Na}_{2} \mathrm{SO}_{4}$. Concentration of the solvent in vacuo afforded a residue, which was purified by column chromatography (hexane-ethyl acetate, 4:1) to give 9 (8.90 g, $88 \%)$ as a colorless oil: ${ }^{1} \mathrm{H}$ NMR $\left(250 \mathrm{MHz}, \mathrm{CDCl}_{3}\right) \delta 3.51(3 \mathrm{H}, \mathrm{s}), 3.86(3 \mathrm{H}, \mathrm{s}), 5.21(2 \mathrm{H}, \mathrm{s}), 6.76$ $(1 \mathrm{H}, \mathrm{d}, J=8.7 \mathrm{~Hz}), 7.10$ (1H, dd, J=2.4, $8.7 \mathrm{~Hz}), 7.30$ (1H, d, J=2.4 Hz); IR (neat) 2999, 2957, 2905, 2835, 1587, 1502, 1462, 1388, 1321, 1298, 1253, 1224, 1199, 1178, 1155, 1132, 1082, 1028, 991, 923, $850,800,763,621 \mathrm{~cm}^{-1}$; EIMS m/z $248\left[(\mathrm{M}+2)^{+},{ }^{81} \mathrm{Br}\right], 246\left(\mathrm{M}^{+},{ }^{79} \mathrm{Br}\right)$. 